\title{
Tensor Norms and the Classical Communication Complexity of Nonlocal Quantum Measurement 1
}

\author{
Yaoyun Shi and Yufan Zhu \\ Department of Electrical Engineering and Computer Science \\ University of Michigan \\ 1301 Beal Avenue \\ Ann Arbor, MI 48109-2122, USA \\ Email: \{shiyy|yufanzhu\}@eecs.umich.edu
}

\begin{abstract}
We initiate the study of quantifying nonlocalness of a bipartite measurement by the minimum amount of classical communication required to simulate the measurement. We derive general upper bounds, which are expressed in terms of certain tensor norms of the measurement operator. As applications, we show that (a) If the amount of communication is constant, quantum and classical communication protocols with unlimited amount of shared entanglement or shared randomness compute the same set of functions; (b) A local hidden variable model needs only a constant amount of communication to create, within an arbitrarily small statistical distance, a distribution resulted from local measurements of an entangled quantum state, as long as the number of measurement outcomes is constant.
\end{abstract}

Keywords: Quantum entanglement, classical simulation, communication complexity, tensor norms, Bell Inequality

\footnotetext{
${ }^{1} \mathrm{~A}$ preliminary version of this paper appeared as part of an article in Proceedings of the the 37th ACM Symposium on Theory of Computing (STOC 2005), 460-467, 2005.
} 


\section{Introduction and summary of results}

Background. Although Einstein himself made significant contributions to the development of quantum mechanics, he famously questioned the "completeness" of the theory with a "paradox" that he formulated with Podolsky and Rosen [16]. Following Bohm [6], the essence of the paradox is: two "quantum coins", possessed by two parties Alice and Bob, may be correlated in a state that can be schematically represented as

$$
\left.\left.\frac{1}{\sqrt{2}}\left(|\operatorname{Head}\rangle_{A} \mid \text { Tail }\right\rangle_{B}-\mid \text { Tail }\right\rangle_{A}|\operatorname{Head}\rangle_{B}\right)
$$

If each party measures his or her coin, with $1 / 2$ probability, one of the two outcomes would be observed. However, once a measurement is made by one party, say, Alice, then Bob would always observe the opposite outcome with certainty. A unique property of the state is that, no matter what property of the coins is measured - be it determining their positions or the velocities - Bob's outcome is also opposite to that of Alice with certainty. Since what Alice does locally should not affect Bob's world, this is at odds with the "uncertainty" principle of quantum mechanics that not all pairs of properties can be determined with certainty.

The Einstein-Podolsky-Rosen (EPR) paradox did not reduce quantum mechanics to contradictions. Instead, it revealed the essence - quantum entanglement - that underlies the many counter-intuitive properties and marvelous capabilities of quantum information. For example, in his far reaching paper [3], John Bell formulated a set of inequalities, referred to as Bell Inequalities now, that must be satisfied by the correlations produced by any so called hidden variable classical model but would nevertheless be violated by some quantum correlations. The latter has been confirmed by several experiments (e.g., [37]). Another seminal example is the quantum key distribution protocol [4, which has been shown to be information theoretically secure [25, 27], as a consequence of properties of quantum entanglement.

Given its importance, quantum entanglement has been the subject of numerous studies (see, e.g., the books [30, 31]). The focus has been on understanding the inherent quantitative tradeoffs among various resources involved in the creation and conversion of entangled states. As entanglement is the result of nonlocal quantum interactions, understanding various aspects of the nonlocality of quantum operations is also of fundamental importance. Quantifying nonlocality of quantum operators is precisely the purpose of this paper.

A natural nonlocality measure of a quantum operation is its generating capacity, which is the maximum entanglement increase that it could create (see e.g., [5]). Another approach, more from a computational point of view, is to consider the amount of resources, such as the time in the case of using elementary Hamiltonians, or the number of elementary gates, required to simulate the operator (e.g., [10, 11]).

Main result. In this paper, we take a completely different approach to quantify the nonlocality of quantum operations, following intuitions from the subject of communication complexity. Our work is not the first to apply communication complexity to the study of 
entanglement. There has been a line of research, which we will review shortly, that studies the classical communication complexity of simulating quantum correlations. Nevertheless, our emphasis is on quantum operators, and we focus on measurement operators, while our approach can be extended to the most general quantum operations.

Consider the following quantum process. Alice and Bob share a bipartite state $|E\rangle_{A B}$. They apply local operations $R_{A}$ and $R_{B}$ to his/her system, before a final measurement $Q$ is applied to the joint system, producing a distribution $\mu=\mu\left(Q,|E\rangle, R_{A}, R_{B}\right)$ of measurement outcomes.

Imagine now that Alice and Bob loose their quantum power. They both know classical descriptions of $Q$ and $|E\rangle$, and that of their local operations, but do not know what the other party's local operation is. From those classical information, they hope to simulate the quantum process, by producing an output whose distribution is close to $\mu$, through a communication process that starts with an unlimited supply of common random bits. We define the classical communication complexity of $Q$, denoted by $\operatorname{Com}(Q)$, to be the minimum number of bits that need to be exchanged by the simulating communication process.

Intuitively, $\operatorname{Com}(Q)$ reflects how nonlocal $Q$ is. Consider, for example, the simple case that $Q$ consists of local operations. If there is no quantum correlation in the initial state, it is clear that Alice and Bob could simulate the quantum process without interaction. We shall see that even if the initial state is entangled, they do need only exchange a constant number of bits.

On the other hand, $\operatorname{Com}(Q)$ could be much larger. Let $n \geq 1$ be an integer. Consider the following operator.

$$
\mathrm{IP}_{n} \stackrel{\text { def }}{=} \sum_{x, y \in\{0,1\}^{n}, x \cdot y=1}|x\rangle\langle x|\otimes| y\rangle\langle y| .
$$

When $R_{A}$ is to create a state $|x\rangle, x \in\{0,1\}^{n}$, and $R_{B}$ creates $|y\rangle, y \in\{0,1\}^{n}$, then $(I P)_{n}$ determines if $x \cdot y=1$. This is the so called "Inner Product" function well studied in the communication complexity literature. It is well known that any classical communication protocol for solving Inner Product requires $\Omega(n)$ bits of communication. In fact, Cleve, van Dam, Nielsen, and Tapp [13] proved that $\Omega(n)$ quantum bits are necessary, too. Thus $\operatorname{Com}(\mathrm{IP})=\Omega(n)$. We do not know if this bound for Com(IP) is tight.

The goal of this paper is to understand how $\operatorname{Com}(Q)$ is determined in general. It is not immediately clear if $\operatorname{Com}(Q)$ can be bounded from above for all $Q$, as the dimension of the initial state $|E\rangle$ could be arbitrarily large. Our main result is to derive a general upper bound on $\operatorname{Com}(Q)$ in terms of a certain operator norm $\|Q\|_{\diamond}$ on $Q$, which is bounded from above polynomially in $Q$ 's dimension.

Theorem 1.1 (Informally). For any bipartite quantum measurement $Q, \operatorname{Com}(Q)=O\left(\|Q\|_{\diamond}^{2}\right)$. In particular, if $K$ is the dimension of the space that $Q$ acts on, $\operatorname{Com}(Q)=O\left(K^{4}\right)$.

The diamond norm $\|Q\|_{\diamond}$ is originally defined on superoperators, and has been a powerful tool in the study of quantum interactive proof systems [20] and quantum circuits on mixed 
states [1]. We make use a natural mapping from bipartite operators to superoperators to define norms on the former based on norms on the latter.

The approach in proving Theorem 1.1 can be extended to obtain general upper bounds on $\operatorname{Com}(Q)$ in terms of other operators norms. Those norms belong to so called tensor norms, i.e., norms $\|\cdot\|_{\alpha}$ that satisfies $\|P\|_{\alpha} \leq\|A\| \cdot\|B\|$, whenever $P=A \otimes B$. Tensor norms have been studied for decades with a great deal of rich concepts and deep results (see, e.g., [15]). In recent years, they have been applied to quantum information theory to characterize and quantify the nonlocality of quantum states [34, 35]. The tensor norms that appear in our upper bounds capture the nonlocality of bipartite operators in their own way, and may have further applications.

Applications on quantum communication complexity. After obtaining those general upper bounds, we show that they in turn have useful applications on quantum communication complexity. Recall that in the setting of communication complexity [40, 41], Alice and Bob wish to compute a function $f(x, y)$, where $x$ is known to Alice only, and $y$ is known only to Bob. The communication complexity of $f$ is the minimum amount of information that Alice and Bob need to exchange in order to compute $f$ correctly for any input. Communication complexity has been a major research field (see, e.g., the book [24]), with many problems of rich structures and deep connections to other aspects of complexity theory.

A concrete application of our result is on the advantage of sharing entanglement in quantum protocols, a question that has puzzled many researchers [12, 8, 22, 28]. It is known that sharing entanglement could give a constant additive advantage [12, 8], or save a half of the communication [13. However, little is known on the limit of the advantage. This is in sharp contrast with the classical case of sharing randomness, where we know that it can only save at most a logarithmic additive term [29]. If there is a quantum protocol that exchanges $q$ qubits with $m$ qubits of prior entanglement, then the best classical simulation we know is $\exp (\Omega(q+m))$. This is embarrassingly large, especially when $q<<m$. Using our upper bound on the classical communication complexity of nonlocal operators, we prove the following result. Note that in the Simultaneous Message Passing (SMP) model with shared randomness, the two parties holding the inputs share an arbitrarily long random string, and each send a single message to a third party, who is required to determine the outcome correctly with high probability.

Theorem 1.2. If a twoway quantum protocol uses q qubits of communication and $m$ qubits of share entanglement, then it can be simulated by a classical protocol using $\exp (O(q))$ bits with shared randomness. The simulation does not depend on $m$. Furthermore, it can be carried out in the SMP model with with shared randomness.

Notice that the exponential dependence on $q$ can not be improved, because of the existence of an exponential separation of quantum and classical communication complexities for some partial function, discovered by Raz [32]. As a consequence of the above theorem, 
Corollary 1.3. If a communication complexity problem has a constant cost quantum communication protocol with shared entanglement, it also has a constant cost classical protocol with shared randomness.

It is interesting to contrast the above with a recent result by Yao [42], which is of a similar type but of the opposite direction.

Theorem 1.4 ([42]). If a communication complexity problem of input size $n$ has a constant cost classical SMP protocol with shared randomness, it has an $O(\log n)$ cost quantum SMP protocol without shared entanglement.

Combining this result with ours, we have

Corollary 1.5. If a communication complexity problem of input size $n$ has a constant cost twoway quantum protocol with shared entanglement, it has an $O(\log n)$ cost quantum $S M P$ protocol without shared entanglement.

Applications on simulating quantum correlations. Yet another application of our classical simulation of quantum measurements is to give efficient simulations of quantum correlations by the hidden variable model assisted with classical communication. The scenario is as follows. Suppose Alice and Bob are given an entangled quantum state. Then each of them, without any communication, applies to their portion of the state some local measurement not known to the other party. The result is a correlated joint distribution on both measurement outcomes. There are such correlations that violate the Bell Inequalities, hence impossible to generate by any reasonable classical procedure in which Alice and Bob do not communicate.

A natural next step to extend the above work of Bell is to investigate the minimum amount of classical communication required to simulate a quantum correlation. Most of the works addressing this question focus on the exact simulation and on measuring a constant number of qubits [39, 2, 14, 36, 7, 26. We study the approximate and asymptotic simulation of quantum correlations, where the joint random variables take a constant number of possible values but are nevertheless produced from (the two party) sharing an entangled state of an arbitrary dimension and applying arbitrary local measurements.

Theorem 1.6 (Informally). In the above scenario, a $O\left(\ln \frac{1}{\epsilon} / \epsilon^{2}\right)$ number of classical bits is sufficient to approximate the quantum correlation with a $\epsilon$ statistical distance.

Organization. The rest of the paper is organized as follows. We start with the description of a general framework for classical simulation of quantum protocols. The cost parameter of this framework is then optimized in the next section, giving the main theorem. In the section that follows we give applications of the theorem. Finally we conclude with several open problems. 


\section{A simulation framework}

Our classical simulation of quantum protocols falls into the following framework. Let $p$ be the acceptance probability (i.e., the probability of outputting 1) of a given quantum protocol (which arises either from a communication task or from a bipartite measurement). We express $p=\left\langle\psi_{A} \mid \psi_{B}\right\rangle$, for two vectors $\left|\psi_{A}\right\rangle$ and $\left|\psi_{B}\right\rangle$ that can be prepared by Alice and Bob by herself/himself. Note that the lengths of the two vectors may be very large, in general. Indeed the shorter their lengths are, the better our simulation is.

More precisely, if for some number $C, \|\left|\psi_{A}\right\rangle \| \leq C$ and $\|\left|\psi_{B}\right\rangle \| \leq C$, then the following simulation uses $O\left(C^{4}\right)$ bits. Alice and Bob send Charlie $\|\left|\psi_{A}\right\rangle \|$ and $\|\left|\psi_{B}\right\rangle \|$, respectively, up to $O(1 / C)$ precision. This requires $O(\log C)$ bits. They then proceed to estimate $\cos \theta$, for the angle $\theta$ between $\left|\psi_{A}\right\rangle$ and $\left|\psi_{B}\right\rangle$ up to a precision of $O\left(1 / C^{2}\right)$. The protocol in Kremer, Nisan and Ron [23], which is based on the following observation of Goemans and Williamson [18, gives a protocol that accomplishes the latter task using $O\left(C^{4}\right)$ bits.

Assume for simplicity that all vectors are real (the complex number case can be easily reduced to the real case). If $|\psi\rangle$ is a random unit vector in the same space of $\left|\phi_{A}\right\rangle$ and $\left|\phi_{B}\right\rangle$, then

$$
\operatorname{Prob}\left[\operatorname{sign}\left(\left\langle\psi \mid \psi_{A}\right\rangle\right) \neq \operatorname{sign}\left(\left\langle\psi \mid \psi_{B}\right\rangle\right)\right]=\theta / \pi .
$$

Hence, in order to estimate $\cos \theta$ with error term $\delta$, it suffices to estimate $\theta / \pi$ to some error term $O(\delta)$ using the above equality checking of signs. Obviously this can be done by a SMP protocol, and by a simple application of Chernoff Bound, requires $O\left(\ln \frac{1}{\epsilon} / \delta^{2}\right)$ repetitions. With $\delta=O\left(\epsilon / C^{2}\right)$, this is $O\left(C^{4} \ln \frac{1}{\epsilon} / \epsilon^{2}\right)$ bits.

We note that 38 , gives a procedure along the lines of checking equality of signs but it produces a random \pm 1 variable whose expectation is precisely $\cos \theta$, though this is not asymptotically advantageous.

We summarize the above discussion as the basis for our future discussions.

Theorem 2.1 ([23, [18]). Suppose the acceptance probability of a quantum protocol can be expressed as $\left\langle\psi_{A} \mid \psi_{B}\right\rangle$, where $\left|\psi_{A}\right\rangle$ and $\left|\psi_{B}\right\rangle$ can be prepared by each party individually. Furthermore, for some number $C, \|\left|\psi_{A}\right\rangle \| \leq C$, and $\|\left|\psi_{B}\right\rangle \| \leq C$. Then there is a classical $S M P$ protocol with shared coins that uses $O\left(C^{4} \ln \frac{1}{\epsilon} / \epsilon^{2}\right)$ bits and whose acceptance probability deviates from that of the protocol by at most $\epsilon$.

\section{The main theorem}

In this section, we formally define the classical communication complexity and the diamond norm of bipartite quantum operators, and derive an upper bound on the former in terms of the latter. We shall focus on the following case: that the measurement gives two outcomes, and that the dimensions of the two systems are the same. Our results can be extended trivially to more general cases. 
We use script letters $\mathcal{N}, \mathcal{M}, \mathcal{F}, \cdots$, to denote Hilbert spaces, and $\mathbf{L}(\mathcal{N})$ to denote the space of operators on $\mathcal{N}$. The identity operator on $\mathcal{N}$ is denoted by $I_{\mathcal{N}}$, and the identity superoperator on $\mathbf{L}(\mathcal{N})$ is denoted by $\mathbf{I}_{\mathcal{N}}$. Recall that a positive-operator-valued measurement (POVM) on a Hilbert space $\mathcal{H}$ is a set of positive semidefinite operators $\left\{Q_{1}, Q_{2}, \cdots, Q_{m}\right\}$ on $\mathcal{H}$, such that $\sum_{i=1}^{m} Q_{i}=I_{\mathcal{H}}$. Each $Q_{i}$ is called a measurement element, and corresponds to the measurement outcome $i$. We may refer to a semidefinite operator $Q$, $0 \leq Q \leq 1$, as a measurement element of the implicit binary POVM $\{Q, I-Q\}$. For more details on the foundations of quantum information processing, refer to the textbook [30].

Classical simulation of quantum measurements. In this subsection we define the central concept of this paper: the classical communication complexity of quantum measurements.

Let $Q$ be measurement element acting on a bipartite system $A B$. Let $|E\rangle_{A^{\prime} B^{\prime}}$ be a bipartite state, where $A^{\prime}\left(B^{\prime}\right)$ includes $A(B)$ as a subsystem. Let $R_{A}$ and $R_{B}$ be physically realizable operators acting on system $A^{\prime}$ and $B^{\prime}$, respectively. Denote by $\mu\left(Q,|E\rangle, R_{A}, R_{B}\right)$ the probability

$$
\mu\left(Q,|E\rangle, R_{A}, R_{B}\right) \stackrel{\text { def }}{=} \operatorname{tr}\left(Q R_{A} \otimes R_{B}(|E\rangle\langle E|)\right) .
$$

Definition 3.1. Let $\epsilon \in[0,1 / 2)$, and $Q$ be a measurement elements. The classical communication complexity of $Q$ with precision $\epsilon$, denoted by $\operatorname{Com}_{\epsilon}(Q)$, is the minimum number $k$ such that for any $|E\rangle, R_{A}$ and $R_{B}$ described above, there is a classical communication protocol between two parties Alice and Bob that satisfies the following conditions:

1. The input of Alice (Bob) is a classical description of $|E\rangle$, and a classical description of $R_{A}\left(R_{B}\right)$;

2. The output is a random binary variable of which the expected value $p$ satisfies

$$
\mid p-\mu\left(Q,|E\rangle, R_{A}, R_{B}\right) \mid \leq \epsilon .
$$

3. The protocol exchanges $\leq k$ bits and is allowed to use an unlimited amount of shared randomness.

The diamond norm on bipartite operators. Let $\mathcal{N}$ be a Hilbert space and $T: \mathbf{L}(\mathcal{N}) \rightarrow$ $\mathbf{L}(\mathcal{N})$ be a superoperator. The diamond norm on super operators is defined as (c.f. [21])

$$
\|T\|_{\diamond} \stackrel{\text { def }}{=} \inf \left\{\|A\|\|B\|: \operatorname{tr}_{\mathcal{F}}\left(A \cdot B^{\dagger}\right)=T, A, B \in \mathbf{L}(\mathcal{N}, \mathcal{N} \otimes \mathcal{F})\right\} .
$$

For our application, the following alternative characterization of the diamond norm is more convenient.

Lemma 3.2 (e.g., [21]). For any superoperator $T$,

$$
\|T\|_{\diamond}=\min \left\{\sqrt{\left\|\sum_{t} A_{t}^{\dagger} A_{t}\right\|} \cdot \sqrt{\left\|\sum_{t} B_{t}^{\dagger} B_{t}\right\|}: \quad A_{t}, B_{t} \in \mathbf{L}(\mathcal{N}), T=\sum_{t} A_{t} \cdot B_{t}^{\dagger}\right\} .
$$


Let $\mathcal{N}_{A}, \mathcal{N}_{B}$, and $\mathcal{N}$ be Hilbert spaces of the same dimension. We fix an isomorphism between any two of them. For an operator in one space, we use the same notation for its images and preimages, under the isomorphisms, in the other spaces.

Let $Q \in \mathbf{L}\left(\mathcal{N}_{A} \otimes \mathcal{N}_{B}\right)$ be a bipartite operator and $Q=\sum_{t} A_{t} \otimes B_{t}^{\dagger}$, for some $A_{t} \in$ $\mathbf{L}\left(\mathcal{N}_{A}\right)$, and $B_{t} \in \mathbf{L}\left(\mathcal{N}_{B}\right)$. Define a mapping $\mathcal{T}$ from bipartite operators on $\mathcal{N}_{A} \otimes \mathcal{N}_{B}$ to superoperators $\mathbf{L}(\mathcal{N}) \rightarrow \mathbf{L}(\mathcal{N})$ by mapping $Q \mapsto \mathcal{T}(Q) \stackrel{\text { def }}{=} \sum_{t} A_{t} \cdot B_{t}^{\dagger}$. It can be easily verified that the mapping is independent of the choice of the decomposition of $Q$ and is indeed an isomorphism.

Definition 3.3. Let $Q \in \mathbf{L}\left(\mathcal{N}_{A} \otimes \mathcal{N}_{B}\right)$ be a bipartite operator. The diamond norm of $Q$, denoted by $\|Q\|_{\diamond}$, is $\|Q\|_{\diamond} \stackrel{\text { def }}{=}\|\mathcal{T}(Q)\|_{\diamond}$.

By Lemma 3.2 , for any $Q$,

$\|Q\|_{\diamond}=\min \left\{\sqrt{\left\|\sum_{t} A_{t}^{\dagger} A_{t}\right\|} \cdot \sqrt{\left\|\sum_{t} B_{t}^{\dagger} B_{t}\right\|}: A_{t} \in \mathbf{L}\left(\mathcal{N}_{A}\right), B_{t} \in \mathbf{L}\left(\mathcal{N}_{B}\right), Q=\sum_{t} A_{t} \otimes B_{t}^{\dagger}\right\}$.

Note that if a superoperator $T=A \cdot B$ for some $A, B \in \mathbf{L}(\mathcal{N}),\|T\|_{\diamond}=\|A\| \cdot\|B\|$. Therefore the diamond norm on bipartite operators is a tensor norm:

Lemma 3.4. If $K=A \otimes B,\|K\|_{\diamond}=\|A\| \cdot\|B\|$.

A nice property of the superoperator diamond norm is that it is "stable", i.e., it remains unchanged when tensored with the identity operator on an additional space [21].

Lemma 3.5. Let $\mathcal{N}, \mathcal{M}$, and $\mathcal{F}$ be Hilbert spaces, and $T: \mathbf{L}(\mathcal{N}) \rightarrow \mathbf{L}(\mathcal{M})$ be a superoperator. Then $\left\|\mathbf{I}_{\mathcal{F}} \otimes T\right\|_{\diamond}=\|T\|_{\diamond}$.

This stability property carries over to our diamond norm and is important for our applications. Let $\mathcal{F}_{A}$ and $\mathcal{F}_{B}$ be Hilbert spaces of the same dimension, and $Q \in \mathbf{L}\left(\mathcal{N}_{A} \otimes \mathcal{N}_{B}\right)$. Denote by $Q_{\mathcal{F}_{A}, \mathcal{F}_{B}}$ the bipartite operator $Q \otimes I_{\mathcal{F}_{A} \otimes \mathcal{F}_{B}}$, where the two subsystems are $\mathcal{N}_{A} \otimes \mathcal{F}_{A}$ and $\mathcal{N}_{B} \otimes \mathcal{F}_{B}$.

Lemma 3.6. For any $Q,\left\|Q_{\mathcal{F}_{A}, \mathcal{F}_{B}}\right\|_{\diamond}=\|Q\|_{\diamond}$.

If $Q$ is a measurement element of a POVM acting on a Hilbert space of dimension $K$, a trivial upper bound on $\|Q\|_{\diamond}$ is $K^{2}$, as each entry of the matrix of $Q$ under any orthonormal basis has a modulus bounded by 1 .

Proposition 3.7. If a bipartite operator $Q$ is measurement element of a POVM acting on a Hilbert space of dimension $K$, then $\|Q\|_{\diamond} \leq K^{2}$.

This bound is not far from being optimal for $\mathrm{IP}_{n}$, in which case $K=2^{2 n}$. To prove a lower bound on $\left\|\mathrm{IP}_{n}\right\|_{\diamond}$, we use a remarkable dual characterization of the diamond norm (e.g., [21, Theorem 11.1). Let $T: L(\mathcal{N}) \rightarrow L(\mathcal{N})$ be superoperator and $\mathcal{G}$ be a space of the same dimension as $\mathcal{N}$. Then

$$
\|T\|_{\diamond}=\sup _{\rho \in L(\mathcal{N} \otimes \mathcal{G}), \rho \neq 0} \frac{\left\|\left(T \otimes \mathbf{I}_{\mathcal{G}}\right)(\rho)\right\|_{\mathrm{tr}}}{\|\rho\|_{\mathrm{tr}}} .
$$


Proposition 3.8. For the $\mathrm{IP}_{n}$ operator defined in Equation 1, $\left\|\mathrm{IP}_{n}\right\|_{\diamond} \| \geq 2^{n-1}$.

Proof. By definition,

$$
\mathcal{T}\left(\mathrm{IP}_{n}\right)=\sum_{x, y \in\{0,1\}^{n}, x \cdot y=1}|x\rangle\langle x|\cdot| y\rangle\langle y| .
$$

We set $\rho=\sum_{x, y}|x\rangle\langle y| \otimes I_{\mathcal{G}}$ in Equation 3, resulting in

$$
\left\|\mathcal{T}\left(\mathrm{IP}_{n}\right)\right\|_{\diamond} \geq \frac{1}{2^{n}} \| \sum_{x, y \in\{0,1\}^{n}, x \cdot y=1}|x\rangle\langle y| \|_{\operatorname{tr}} .
$$

The right-hand-side is at least

$$
\frac{1}{2^{n}} \operatorname{trace}\left(\sum_{x, y \in\{0,1\}^{n}, x \cdot y=1}|x\rangle\langle y|\right) \geq 2^{n-1} .
$$

Thus $\left\|\operatorname{IP}_{n}\right\|_{\diamond} \geq 2^{n-1}$.

We conclude this subsection by noting that our diamond norm on bipartite operators appears natural in connection with the following matrix analogy of the Cauchy Schwartz Inequality.

Theorem 3.9 (Jocić [19]). For any operators $A_{t}$ and $B_{t}$,

$$
\left\|\sum_{t} A_{t} \otimes B_{t}^{\dagger}\right\| \leq \sqrt{\left\|\sum_{t} A_{t}^{\dagger} A_{t}\right\|} \cdot \sqrt{\left\|\sum_{t} B_{t}^{\dagger} B_{t}\right\|} .
$$

Hence, if $\|Q\|_{\diamond}$ is precisely the smallest right-hand-side when $A_{t}$ and $B_{t}$ are such that $Q=\sum_{t} A_{t} \otimes B_{t}^{\dagger}$. Inequality (4) may actually be proved by the same approach that we use to prove Theorem 3.10 below.

Upper bounding $\operatorname{Com}(Q)$ by the diamond norm. We now use the diamond norm to derive an upper bound on $\operatorname{Com}_{\epsilon}(Q)$. Recall that if $\mathcal{M}$ and $\mathcal{N}$ are two Hilbert spaces, an isometric embedding $U: \mathcal{M} \rightarrow \mathcal{N}$ is a linear map that satisfies $U^{\dagger} U=I_{\mathcal{M}}$.

Theorem 3.10. For any bipartite positive semidefinite operator $Q$ acting on a Hilbert space of dimension $K$,

$$
\operatorname{Com}_{\epsilon}(Q)=O\left(\|Q\|_{\diamond}^{2} \cdot \ln \frac{1}{\epsilon} / \epsilon^{2}\right)
$$

In particular $\operatorname{Com}_{\epsilon}(Q)=O\left(K^{4} \log \frac{1}{\epsilon} / \epsilon^{2}\right)$. Furthermore, the upper bound (5) can be achieved by a SMP protocol with shared randomness.

Proof. Without loss of generality, assume that on receiving their portions of $|E\rangle$, Alice and Bob apply an isometric embedding $U: \mathcal{M}_{A} \rightarrow \mathcal{N}_{A} \otimes \mathcal{F}_{A}$, and $V: \mathcal{M}_{B} \rightarrow \mathcal{N}_{B} \otimes \mathcal{F}_{B}$, respectively, for some Hilbert spaces $\mathcal{F}_{A}$ and $\mathcal{F}_{B}$ with an equal dimension. The distribution 
resulted from Charlie's measuring $Q$ on $\operatorname{Tr}_{\mathcal{F}_{A}, \mathcal{F}_{B}}\left((U \otimes V)|E\rangle\langle E|(U \otimes V)^{\dagger}\right)$ is the same as that of Charlie applying $Q_{\mathcal{F}_{A}, \mathcal{F}_{B}}$ on the larger state $(U \otimes V)|E\rangle\langle E|(U \otimes V)^{\dagger}$. By Lemma 3.6, $\left\|Q_{\mathcal{F}_{A}, \mathcal{F}_{B}}\right\|_{\diamond}=\|Q\|_{\diamond}$. Therefore, to prove the theorem we need only to consider isometric embeddings $U: \mathcal{M}_{A} \rightarrow \mathcal{N}_{A}$ and $V: \mathcal{M}_{A} \rightarrow \mathcal{N}_{B}$.

Without loss of generality, we assume that Alice and Bob have agreed on a Schmidt decomposition $|E\rangle=\sum_{i} \sqrt{p_{i}}|i\rangle_{A} \otimes|i\rangle_{B}$, for some $p_{i} \geq 0, \sum_{i} p_{i}=1$, and for an orthonormal basis $\{|i\rangle\}$. Denote by $\left|i_{A}\right\rangle \stackrel{\text { def }}{=} U|i\rangle$, and $\left|i_{B}\right\rangle \stackrel{\text { def }}{=} V|i\rangle$. Then the message that Charlie receives is $|\bar{E}\rangle \stackrel{\text { def }}{=}(U \otimes V)|E\rangle=\sum_{i} \sqrt{p_{i}}\left|i_{A}\right\rangle \otimes\left|i_{B}\right\rangle$.

Suppose $\|Q\|_{\diamond}$ is achieved under the decomposition $Q=\sum_{t} A_{t} \otimes B_{t}^{\dagger}$, with which if $Q_{A} \stackrel{\text { def }}{=} \sum_{t} A_{t}^{\dagger} A_{t}$, and, $Q_{B} \stackrel{\text { def }}{=} \sum_{t} B_{t}^{\dagger} B_{t}$, we have $\left\|Q_{A}\right\|=\left\|Q_{B}\right\|=\|Q\|_{\diamond}$. With those definitions, we have

$$
p=\langle\bar{E}|Q| \bar{E}\rangle=\sum_{i, j, t} \sqrt{p_{i} p_{j}}\left\langle i_{A}\left|A_{t}\right| j_{A}\right\rangle \cdot\left\langle i_{B}\left|B_{t}^{\dagger}\right| j_{B}\right\rangle
$$

Define two vectors

$$
\begin{gathered}
\left|\psi_{A}\right\rangle=\sum_{i, j, t} \sqrt{p_{j}}\left\langle j_{A}\left|A_{t}^{\dagger}\right| i_{A}\right\rangle|i, j, t\rangle, \quad \text { and }, \\
\left|\psi_{B}\right\rangle=\sum_{i, j, t} \sqrt{p_{i}}\left\langle i_{B}\left|B_{t}^{\dagger}\right| j_{B}\right\rangle|i, j, t\rangle .
\end{gathered}
$$

Then $p=\left\langle\psi_{A} \mid \psi_{B}\right\rangle$. Further, with $\rho_{A} \stackrel{\text { def }}{=} \sum_{j} p_{j}\left|j_{A}\right\rangle\left\langle j_{A}\right|$,

$$
\left\langle\psi_{A} \mid \psi_{A}\right\rangle=\sum_{i, j, t} p_{j}\left|\left\langle j_{A}\left|A_{t}^{\dagger}\right| i_{A}\right\rangle\right|^{2}=\operatorname{tr}\left(\rho_{A} Q_{A}\right) \leq\left\|Q_{A}\right\|=\|Q\|_{\diamond}
$$

Similarly, $\left\langle\psi_{B} \mid \psi_{B}\right\rangle \leq\left\|Q_{B}\right\|=\|Q\|_{\diamond}$. Therefore, by Theorem 2.1, the measurement scenario can be approximated by a classical SMP with shared coins to be within an $\epsilon$ precision using $O\left(\|Q\|_{\diamond}^{2} \ln \frac{1}{\epsilon} / \epsilon^{2}\right)$ bits. This bound is $O\left(K^{4} \log \frac{1}{\epsilon} / \epsilon^{2}\right)$ as $\|Q\|_{\diamond}=O\left(K^{2}\right)$ by Proposition 3.7 .

Remark 3.11. One may improve the above upper bound on $\operatorname{Com}_{\epsilon}(Q)$ by a more carefully chosen $\left|\psi_{A}\right\rangle$ and $\left|\psi_{B}\right\rangle$ in Equation 6 and 7 . More specifically, let $\alpha \in[0,1]$, define

$$
\begin{gathered}
\left|\psi_{A}^{\alpha}\right\rangle=\sum_{i, j, t} \sqrt{p_{i}^{\alpha} p_{j}^{1-\alpha}}\left\langle j_{A}\left|A_{t}^{\dagger}\right| i_{A}\right\rangle|i, j, t\rangle, \quad \text { and }, \\
\left|\psi_{B}^{\alpha}\right\rangle=\sum_{i, j, t} \sqrt{p_{i}^{1-\alpha} p_{j}^{\alpha}}\left\langle i_{B}\left|B_{t}^{\dagger}\right| j_{B}\right\rangle|i, j, t\rangle .
\end{gathered}
$$

One can verify that minimizing $\|\left|\psi_{A}\right\rangle\|\cdot\|\left|\psi_{B}\right\rangle \|$ over all decompositions of $Q$ gives rise to a tensor norm, which we do not know if is stable under tensoring with identity superoperators. Although we have not found any useful application of an $\alpha \neq 0$, we cannot rule out the possibility that a carefully chosen $\alpha$ may give a better bound. 
Remark 3.12. In the case that $|E\rangle$ is not entangled, the same approach in Theorem 3.10 can be used to derive a systematic classical simulation. More specifically, in this context we would like to estimate $p=\left\langle\phi_{A} \otimes \phi_{B}|Q| \phi_{A} \otimes \phi_{B}\right\rangle$, for a state $\left|\phi_{A}\right\rangle$ known to Alice only and a state $\left|\phi_{B}\right\rangle$ known to Bob only. For a decomposition of $Q=\sum_{t} A_{t} \otimes B_{t}^{\dagger}$, we define

$$
\left|\psi_{A}\right\rangle=\sum_{t}\left\langle\phi_{A}\left|A_{t}^{\dagger}\right| \phi_{A}\right\rangle|t\rangle, \quad \text { and, } \quad\left|\psi_{B}\right\rangle=\sum_{t}\left\langle\phi_{B}\left|B_{t}^{\dagger}\right| \phi_{B}\right\rangle|t\rangle
$$

Then $p=\left\langle\psi_{A} \mid \psi_{B}\right\rangle$. It can be verified that

$$
\|Q\|_{\otimes} \stackrel{\text { def }}{=} \inf \left\{\left\|\psi_{A}\right\| \cdot\left\|\psi_{B}\right\|: Q=\sum_{t} A_{t} \otimes B_{t}^{\dagger}\right\}
$$

defines a tensor norm and $\|Q\|_{\otimes} \leq\|Q\|_{\diamond}$. This approach gives a constant cost simulation of the elegant quantum fingerprint protocol of Buhrman, Cleve, Watrous, and de Wolf [9] for testing equality of two input strings.

\section{Applications}

We now apply the above to derive classical upper bounds on quantum communication complexity.

Quantum SMP with shared entanglement. If the quantum protocol is in the SMP model with shared entanglement, we immediately have,

Corollary 4.1 (of Theorem 3.10 ). If in a quantum SMP protocol, Charlie applies the measurement $P$, then the protocol can be simulated by a classical SMP protocol with shared coins and using $O\left(\|P\|_{\diamond}^{2}\right)$ bits.

Twoway interactive quantum communication with shared entanglement. Now consider the general twoway interactive quantum communication. We need the following lemma due to Yao [41, and the following formulation is from [33]:

Lemma 4.2 ([41, 33]). Let $\mathcal{P}$ be a two-party interactive quantum communication protocol that uses $q$ qubits. Let $\mathcal{H}_{A}$ and $\mathcal{H}_{B}$ be the state spaces of Alice and Bob, respectively. For an input $(x, y)$, denote by $\left|\Phi_{x, y}\right\rangle_{A B}$ the joint state of Alice, Bob before the protocol starts. Then there exist linear operators $A_{h} \in \mathbf{L}\left(\mathcal{H}_{A}\right)$, and $B_{h} \in \mathbf{L}\left(\mathcal{H}_{B}\right)$, for each $h \in\{0,1\}^{q-1}$, such that

(a) $\left\|A_{h}\right\| \leq 1$ and $\left\|B_{h}\right\| \leq 1$ for all $h \in\{0,1\}^{q-1}$;

(b) the acceptance probability of $\mathcal{P}$ on input $x$ and $y$ is $\| P\left|\Phi_{x, y}\right\rangle \|^{2}$, where $P \stackrel{\text { def }}{=} \sum_{h \in\{0,1\}^{q-1}} A_{h} \otimes$ $B_{h}$.

We are now ready to prove Theorem 1.2 , 
Proof of Theorem 1.2. Let $|E\rangle_{A B}$ be the shared entanglement, For an $n$-bit binary string $x$, denote by $U_{x}$ the isometric embedding from $\mathbb{C}$ to $\mathbb{C}^{\otimes 2^{n}}$ that maps $c \mapsto c|x\rangle$. Let $P, A_{h}$, and $B_{h}$ be those in Lemma 4.2. Then the quantum protocol gives rise to a measurement scenario in which the measurement is $P^{\dagger} P$, the shared entanglement is $|E\rangle$, and on an input pair $(x, y)$, Alice's private operator is $U_{x}$ and that of Bob is $U_{y}$.

By Theorem 3.10, the acceptance probability can be estimated with $O\left(\left\|P^{\dagger} P\right\|_{\diamond}^{2}\right)$ bits of communication in the SMP model with shared randomness. Since $\|\cdot\|_{\diamond}$ is a tensor norm, we have

$$
\left\|P^{\dagger} P\right\|_{\diamond} \leq \sum_{h, h^{\prime}}\left\|\left(\left(A_{h^{\prime}}\right)^{\dagger} A_{h}\right) \otimes\left(\left(B_{h^{\prime}}\right)^{\dagger} B_{h}\right)\right\|_{\diamond}=\sum_{h, h^{\prime}}\left\|A_{h}\right\|\left\|A_{h^{\prime}}\right\|\left\|B_{h}\right\|\left\|B_{h^{\prime}}\right\| \leq 2^{2(q-1)} .
$$

The last inequality is because $\left\|A_{h}\right\| \leq 1$ and $\left\|B_{h}\right\| \leq 1$ for all $h$. Hence the acceptance probability can be estimated by a classical SMP protocol using $\exp (O(q))$ bits.

Corollary 1.3 follows trivially from the above by setting $q$ to be a constant. Corollary 1.5 follows immediately from Theorem 1.4 and Corollary 1.3 together with the following observation.

Lemma 4.3. If a communication complexity problem has a classical twoway protocol with shared randomness and $b$ bits of cost, it has a classical SMP protocol with shared randomness and $O\left(b 2^{b / 2}\right)$ bits of communication.

Proof. Fix a twoway protocol for the problem in which Alice sends $b_{A}$ bits and bob sends $b_{B}$ bits. To simulate this protocol in the SMP model with shared randomness, Alice sends the referee $2^{b_{B}}$ strings each of which has $b_{A}$ bits and is consistent with her input and a string of $b_{B}$ bits interpreted as Bob's messages. Bob applies the same strategy to sends $2^{b_{A}}$ strings of $b_{B}$ bits. The referee is then able to reconstruct a string of $b$ bits, which is precisely the transcript of communication in the original protocol with the same input and random string. Hence by outputting the last bit of the reconstructed message, this SMP protocol achieves the same error probability of the original protocol. The cost of the simulating protocol is $2^{b_{A}} b_{B}+2^{b_{B}} b_{A}=O\left(b 2^{b / 2}\right)$ bits.

Simulating quantum correlations. We shall define precisely what we mean by simulating quantum correlations.

We define a quantum measurement game as a triple $G=\left(|E\rangle_{A B}, \mathcal{P}_{A}, \mathcal{P}_{B}\right)$, where $|E\rangle_{A B}$ is a bipartite quantum state, $\mathcal{P}_{A}, \mathcal{P}_{B}$ are sets of possible measurements on the system $A$ and the system $B$, respectively. Let $\mathcal{V}_{A}\left(\mathcal{V}_{B}\right.$, respectively) be the set of possible measurement outcomes of $\mathcal{P}_{A}\left(\mathcal{P}_{B}\right.$, respectively). For $P_{A} \in \mathcal{P}_{A}$ and $P_{B} \in \mathcal{P}_{B}$, denote by $\omega_{G}\left(P_{A}, P_{B}\right)$ the distribution of the measurement outcomes when $P_{A} \otimes P_{B}$ is applied to $|E\rangle$.

A classical simulation of a quantum measurement game $G=\left(|E\rangle_{A B}, \mathcal{P}_{A}, \mathcal{P}_{B}\right)$ is a classical communication protocol between two parties Alice and Bob, who start with an unlimited mount of shared randomness, and Alice has the classical description of an element $P_{A} \in \mathcal{P}_{A}$, while Bob has the classical description of an element $P_{B} \in \mathcal{P}_{B}$. At the end of the protocol, 
Alice (and Bob) outputs an element from $\mathcal{V}_{A}\left(\mathcal{P}_{B}\right.$, respectively), resulting in a distribution $\tilde{\omega}\left(P_{A}, P_{B}\right)$.

We are now able to rigorously state Theorem 1.6. Recall that the statistical distance between two distributions $\pi=\left(p_{1}, \cdots, p_{n}\right)$ and $\tilde{\pi}=\left(\tilde{p}_{1}, \cdots, \tilde{p}_{n}\right)$ is $\|\pi-\tilde{\pi}\|_{1} \stackrel{\text { def }}{=} \sum_{i}\left|p_{i}-\tilde{p}_{i}\right|$.

Theorem 4.4. Let $G=\left(|E\rangle_{A B}, \mathcal{P}_{A}, \mathcal{P}_{B}\right)$ be a quantum measurement game, $m=\left|\mathcal{V}_{A}\right| \cdot\left|\mathcal{V}_{B}\right|$, and $\epsilon \in \mathbb{R}, 0 \leq \epsilon<1$. There is a classical simulation of $G$ that exchanges $O\left(\frac{m^{3}}{\epsilon^{2}} \cdot \ln \frac{m}{\epsilon}\right)$ number of bits and the output distribution $\tilde{\omega}\left(P_{A}, P_{B}\right)$ for any $P_{A} \in \mathcal{P}_{A}$ and $P_{B} \in \mathcal{P}_{B}$ satisfies

$$
\left\|\tilde{\omega}\left(P_{A}, P_{B}\right)-\omega_{G}\left(P_{A}, P_{B}\right)\right\|_{1} \leq \epsilon .
$$

In particular, the simulation cost is $O\left(\log \frac{1}{\epsilon} / \epsilon^{2}\right)$ if $m=O(1)$.

Proof. Recall that a POVM measurement can be expressed as a physically realizable operator followed by a projective measurement (see, e.g., [21]). Thus we can assume without loss of generality that there exist projections $P_{A}^{v}, v \in \mathcal{V}_{A}$, and $P_{B}^{v^{\prime}}, v^{\prime} \in \mathcal{V}_{B}$, such that for each $P_{A} \in \mathcal{P}_{A}\left(P_{B} \in \mathcal{P}_{B}\right)$, there is an isometric embedding $U_{A}\left(U_{B}\right)$ so that $P_{A}\left(P_{B}\right)$ consists of the measurement elements $\left\{U_{A}^{\dagger} P^{v} U_{A}: v \in \mathcal{V}_{A}\right\}\left(\left\{U_{B}^{\dagger} P^{v^{\prime}} U_{B}: v^{\prime} \in \mathcal{V}_{B}\right\}\right)$.

Fix a pair of measurements $\left(P_{A}, P_{B}\right)$. In the classical simulation protocol, Alice and Bob first compute the probability of outputting $\left(v, v^{\prime}\right)$ to be within $\epsilon / m$ error, for each $v \in \mathcal{V}_{A}$ and $v^{\prime} \in \mathcal{V}_{B}$. They then output $\left(v, v^{\prime}\right)$ according to the probabilities computed. Thus $\tilde{\omega}\left(P_{A}, P_{B}\right)$ is within $\epsilon$ statistical distance to $\omega\left(P_{A}, P_{B}\right)$.

Fix a pair of possible outcome $\left(v, v^{\prime}\right)$. Let $P^{v, v^{\prime}} \stackrel{\text { def }}{=} P_{A}^{v} \otimes P_{B}^{v^{\prime}}$. Then by Lemma 3.4, $\left\|P^{v, v^{\prime}}\right\|_{\diamond}=\left\|P_{A}^{v}\right\| \cdot\left\|P_{A}^{v^{\prime}}\right\| \leq 1$. The estimation of $\omega_{G}\left(P_{A}, P_{B}\right)$ now becomes the simulation of the measurement element $P^{v, v^{\prime}}$ with the initial state being $|E\rangle$, and the local physically realizable operators being $U_{A}^{\dagger} \cdot U_{A}$ and $U_{B}^{\dagger} \cdot U_{B}$.

Hence by Theorem 3.10, the probability of observing outcome $\left(v, v^{\prime}\right)$ can be calculated to be within $O(\epsilon / m)$ precision by by a classical protocol using $O\left(m^{2} \ln (m / \epsilon) / \epsilon^{2}\right)$ bits. Thus the overall simulation cost is $O\left(m^{3} \ln (m / \epsilon) / \epsilon^{2}\right)$ bits, which is $O\left(\log \frac{1}{\epsilon} / \epsilon^{2}\right)$ when $m=O(1)$.

\section{Conclusion and open problems}

A central mission of quantum information theory is to understand quantitatively the boundaries between quantum and classical processes. In this paper, we provide an alternative measure of nonlocalness of bipartite quantum measurements: the minimum amount of classical communication required to simulate the quantum measurement. After defining this concept, we give an upper bound by constructing a simulating protocol. The upper bound is expressed in terms of a tensor norm, which captures nonlocalness in its own way, and may be of independent interest and further applications. Variants of our protocol also lead to variants of the main upper bound in terms of other tensor norms.

We then apply our upper bound to the classical simulation of quantum communication protocols and the construction of local hidden variable models augmented with classical 
communication. In particular, we show that quantum and classical communication protocols with unlimited shared entanglement or randomness compute the same set of functions, if the amount of communication is constant. We also show that local measurements of an entangled state can be simulated by a local hidden variable model with a constant amount of communication, as long as the number of measurement outcomes is constant.

Our study is only the first step toward understanding the classical communication complexity of bipartite measurements. An obvious open problem is to prove or disprove that the bound in Theorem 3.10 is tight. Another basic question is to prove a strong lower bound (exponential in the number of qubits) on $\operatorname{Com}(Q)$ for some $Q$.

It would be interesting to relate $\operatorname{Com}(Q)$ to other measures of nonlocality, such as the entanglement capacity, and the minimum number of elementary gates, or the amount of time for evolving some elementary Hamiltonian, needed to approximate $Q$. It is conceivable that by the comparisons of those measures may lead to a unique and representative measure of nonlocalness.

A recent progress on the question of the usefulness of quantum entanglement was made by Gavinsky [17], in which he showed that entanglement is responsible for exponential savings for some communication tasks and in some restricted models. Whether or not entanglement could result in exponential savings for the more standard two-way communication model and for the computation of functions remains unsolved. Can our result on removing the entanglement be strengthened to that one can always use an amount of entanglement linear in size of the messages, with at most a logarithmic additive term?

The cost of our protocol for simulating quantum correlations depends linearly on the number of measurement outcomes. Is this dependence necessary or can one dramatically reduce it?

Finally, it appears a very promising direction to us to further exploring the connections of tensor norms and nonlocalness of quantum states and operations.

\section{Acknowledgments}

We are indebted to Wei Huang, Amnon Ta-Shma, and the anonymous reviewers for their valuable suggestions on improving the presentation of this paper.

\section{References}

[1] D. Aharonov, A. Kitaev, and N. Nisan. Quantum circuits with mixed states. In Proceedings of the 31th Annual ACM Symposium on the Theory of Computation (STOC), pages 20-30, 1998.

[2] D. Bacon and B. F. Toner. Bell inequalities with auxiliary communication. Phys. Rev. Lett., 90(15):157904, Apr 2003.

[3] J. S. Bell. On the Einstein-Podolsky-Rosen paradox. Physics, 1:195, 1964. 
[4] C. H. Bennett and G. Brassard. Quantum cryptography: Public key distribution and coin tossing. In Proceedings of IEEE international Conference on Computers, Systems and Signal Processing, Bangalore, India, page 175, New York, 1984. IEEE Press.

[5] C. H. Bennett, A. W. Harrow, D. W. Leung, and J. A. Smolin. On the capacities of bipartite Hamiltonians and unitary gates, 2002.

[6] D. Bohm. The paradox of Einstein, Rosen, and Podolsky. In Quantum Theory and Measurement, pages 611-623. Prentice-Hall, 1951.

[7] G. Brassard, R. Cleve, and A. Tapp. Cost of exactly simulating quantum entanglement with classical communication. Phys. Rev. Lett., 83:1874-1877, 1999.

[8] H. Buhrman, R. Cleve, and W. van Dam. Quantum entanglement and communication complexity. SIAM J. Comp., 30(6):1829-1841, March 2001.

[9] H. Buhrman, R. Cleve, J. Watrous, and R. de Wolf. Quantum fingerprinting. Phys. Rev. Lett., 87(16):167902, October 2001.

[10] A. M. Childs, H. L. Haselgrove, and M. A. Nielsen. Lower bounds on the complexity of simulating quantum gates. Phys. Rev. A, 68:052311-052316, 2003.

[11] A. M. Childs, D. W. Leung, F. Verstraete, and G. Vidal. Asymptotic entanglement capacity of the Ising and anisotropic Heisenberg interactions. Quantum Information and Computation, 3:97, 2003.

[12] R. Cleve and H. Buhrman. Substituting quantum entanglement for communication. Phys. Rev. A, 56:1201, 1997.

[13] R. Cleve, W. van Dam, M. Nielsen, and A. Tapp. Quantum entanglement and the communication complexity of the inner product function. Lecture Notes in Computer Science, 1509:61-74, 1999.

[14] J. A. Csirik. Cost of exactly simulating a bell pair using classical communication. Phys. Rev. A, 66(1):014302, Jul 2002.

[15] A. Defant and K. Floret. Tensor norms and operator ideals, volume 176 of NorthHolland Mathematics Studies. North-Holland Publishing Co., Amsterdam, 1993.

[16] A. Einstein, B. Podolsky, and N. Rosen. Can quantum-mechanical description of reality be considered complete? Phys. Rev., 47(10):777-780, May 1935.

[17] D. Gavinsky. On the role of shared entanglement. quant-ph/0604052, 2006.

[18] M. X. Goemans and D. P. Williamson. .879-approximation algorithms for max cut and $\max 2$ sat. In $S T O C$, pages 422-431, 1994. 
[19] D. R. Jocić. The Cauchy-Schwarz norm inequality for elementary operators in Schatten ideals. J. London Math. Soc. (2), 60(3):925-934, 1999.

[20] A. Kitaev and J. Watrous. Parallelization, amplification, and exponential time simulation of quantum interactive proof systems. In Proceedings of the thirty-second annual ACM symposium on Theory of computing, pages 608-617. ACM Press, 2000.

[21] A. Y. Kitaev, A. H. Shen, and M. N. Vyalyi. Classical and quantum computation, volume 47 of Graduate Studies in Mathematics. American Mathematical Society, Providence, RI, 2002. Translated from the 1999 Russian original by Lester J. Senechal.

[22] H. Klauck. Lower bounds for quantum communication complexity. In B. Werner, editor, Proceedings of the 42nd Annual Symposium on Foundations of Computer Science (FOCS-01), pages 288-297, Los Alamitos, CA, Oct. 14-17 2001. IEEE Computer Society.

[23] I. Kremer, N. Nisan, and D. Ron. On randomized one-round communication complexity. In Proceedings of the twenty-seventh annual ACM symposium on Theory of computing, pages 596-605. ACM Press, 1995.

[24] E. Kushilevitz and N. Nisan. Communication Complexity. Cambridge University Press, Cambridge, 1997.

[25] H.-K. Lo and H. F. Chau. Unconditional security of quantum key distribution over arbitrarily long distances. Science, 283(5410):2050-2056, March 1999.

[26] T. Maudlin. In D. Hull, M. Forbes, and K. Okruhlik, editors, PSA, volume 1, pages 404-417. Philosophy of Science Association, 1992.

[27] D. Mayers. Unconditional security in quantum cryptography. Journal of the ACM, 48, 2001.

[28] A. Nayak and J. Salzman. On communication over an entanglement-assisted quantum channel. In Proceedings of the thirty-fourth annual ACM symposium on Theory of computing, pages 698-704. ACM Press, 2002.

[29] I. Newman. Private vs. common random bits in communication complexity. Information Processing Letters, 39(2):67-71, 31 July 1991.

[30] M. A. Nielsen and I. L. Chuang. Quantum Computation and Quantum Information. Cambridge University Press, Cambridge, UK, 2000.

[31] J. Preskill. Lecture notes for physics 229: Quantum information and computation.

[32] R. Raz. Exponential separation of quantum and classical communication complexity. In Proceedings of the Thirty-First Annual ACM Symposium on Theory of Computing 
(STOC'99), pages 358-367, New York, May 1999. Association for Computing Machinery.

[33] A. A. Razborov. Quantum communication complexity of symmetric predicates (Russian). Izvestiya of the Russian Academy of Science, Mathematics, 6, 2002. English translation available at http://genesis.mi.ras.ru/ razborov/qcc_eng.ps.

[34] O. Rudolph. A separability criterion for density operators. J. Phys. A-Math. Gen., 33(21):3951-3955, June 2000.

[35] O. Rudolph. Further results on the cross norm criterion for separability. Preprint available at quant-ph/0202143, February 2002.

[36] M. Steiner. Towards quantifying non-local information transfer: Finite- bit non-locality. Phys. Lett., A270:239-244, 2000.

[37] W. Tittel, J. Brendel, H. Zbinden, and N. Gisin. Violation of Bell Inequalities by photons more than $10 \mathrm{~km}$ apart. Phys. Rev. Lett., 81(17):3563, Oct 1998.

[38] B. Toner and D. Bacon. Communication cost of simulating Bell correlations. Phys Rev Lett., 91(18):187904, Oct 2003.

[39] B. F. Toner and D. Bacon. Communication cost of simulating bell correlations. Phys. Rev. Lett., 91(18):187904, Oct 2003.

[40] A. C.-C. Yao. Some complexity questions related to distributive computing. In Eleventh Annual ACM Symposium on Theory of Computing (STOC 'ry), pages 209-213, New York, Apr. 1979. ACM.

[41] A. C.-C. Yao. Quantum circuit complexity. In 34th Annual Symposium on Foundations of Computer Science: November 3-5, 1993, Palo Alto, California: proceedings [papers], pages 352-361. IEEE Computer Society Press, 1993.

[42] A. C.-C. Yao. On the power of quantum fingerprinting. In ACM, editor, Proceedings of the Thirty-Fifth ACM Symposium on Theory of Computing, San Diego, CA, USA, June 9-11, 2003, pages 77-81, New York, NY, USA, 2003. ACM Press. 